\title{
Correspondence
}

Cerebrovasc Dis 2007;24:480-481

DOI: 10.1159/000108924

\section{Comment on 'Is Transcranial Doppler for the Detection of Venous-to-Arterial Circulation Shunts Reproducible?'}

Vijay K. Sharma, Bernard P.L. Chan

Division of Neurology, National University Hospital, Singapore, Singapore

\section{Dear Sir,}

We read with interest the study by Sastry et al. [1] regarding the detection of venous-to-arterial circulation shunt (v-aCS) by transcranial Doppler (TCD). The paper however, raises some important issues.

Transesophageal echocardiography (TEE) is still considered the gold standard for diagnosing the presence of a right-to-left shunt; however it is poorly tolerated by the patients and requires sedation, thus limiting the patients' ability to perform an adequate Valsalva maneuver. TCD has proven to be a reliable technique and has been reported to be more sensitive and specific than TEE $[2,3]$. A recent study with TCD and TEE performed simultaneously showed an almost perfect concordance in patent foramen ovale detection and right-to-left shunt quantification [4].

Counting the microbubbles in agitated saline is a novel aspect of this paper. One potential limitation of this technique could be selecting the portion of the agitated air-saline-blood mixture for counting under the microscope, since the buoyancy of the artificially generated microbubbles may tend to keep them in the higher segments of the mixture. The difference in their number due to addition of blood and shaking through a 3-way connector may explain the variations in the results and sensitivity of TCD among the previous studies.

The currently recommended position for the detection of $\mathrm{v}$-aCS is the supine position. This is based upon the study by Schwarze et al. [5] that showed microemboli decline from the supine to the sitting position. Telman et al. [6] reported that the sitting position increased the yield of microemboli detection but the results were not statistically significant. Both studies had a small sample size and further investigation appears warranted. The increased number of microbubbles traversing the heart in the sitting position may be related to the anatomy of the patent foramen ovale, which is located anteriorly and superiorly in the right atrium [7]. Thus, the sitting position may cause bubbles to rise faster in the right atrium; because of their buoyancy, increased opening of the shunt flap and enhanced by Valsalva maneuver, more microbubbles may cross the right-to-left shunt.
Finally, v-aCS grading of 'significant', 'large' and 'major' was determined by the number of microbubbles detected on 'singlegate' TCD. The sensitivity of TCD may be enhanced by using power M-mode owing to its overlapping and contiguous multiple gates [8]. Power M-mode TCD produces unique signatures of emboli, appearing as brightly colored embolic tracks as they pass through all the arteries along the ultrasound beam. Since a significantly larger number of microembolic signals are counted by power M-mode TCD, especially in the anterior cerebral arteries, better and quantitative assessment is possible. A higher sensitivity of power M-mode TCD with a quantitative assessment may be an alternative to TEE in diagnosing $\mathrm{v}$-aCS, especially in predicting whether a right-to-left intracardiac shunt is present and can be found by catheterization [9].

We believe that new TCD criteria need to be developed for the diagnosis of $\mathrm{v}-\mathrm{aCS}$. This can be achieved by using power M-mode TCD. Repetitive evaluations in different body positions with controlled proportions of blood, air and saline in addition to a uniform agitation maneuver may help in developing better, reliable and reproducible diagnosis of $\mathrm{v}$-aCS. The findings can initially be validated by performing power M-mode TCD simultaneously with TEE and then using the former as an alternative technique for the detection of $\mathrm{v}$-aCS.

\section{References}

1 Sastry S, Daly K, Chengodu T, McCollum C: Is transcranial Doppler for the detection of venous-to-arterial circulation shunts reproducible? Cerebrovasc Dis 2007;23:424-429.

2 Jauss M, Kaps M, Keberle M, Haberbosch W, Dorndorf W: A comparison of transesophageal echocardiography and transcranial Doppler sonography with contrast medium in the detection of patent foramen ovale. Stroke 1994;25:1265-1267.

3 Job FP, Ringelstein EB, Grafen Y, Flachskampf FA, Doherty C, Stockmanns A, Hanrath P: Comparison of transcranial contrast Doppler sonography and transesophageal contrast echocardiography for the detection of patent foramen ovale in young stroke patients. Am J Cardiol 1994;74:381-384.

4 Belvis R, Leta RG, Marti-Fabregas J, Cocho D, Carreras F, Pons-Llado G, Marti-Vilalta JL: Almost perfect concordance between simultaneous transcranial Doppler and transesophageal echocardiography in the quantification of right-to-left shunts. J Neuroimaging 2006;16:133138.

5 Schwarze JJ, Sander D, Kukla C, Wittich I, Babikian VL, Klingelhofer $\mathrm{J}$ : Methodological parameters influence the detection of right to left shunts by contrast transcranial Doppler ultrasonography. Stroke 1999; 30:1234-1239.

6 Telman G, Kouperberg E, Sprecher E, Yarnitsky D: The position of the patients in the diagnosis of patent foramen ovale by transcranial Doppler. J Neuroimaging 2003;13:356-358.

7 Penter P: Patent foramen ovale: an anatomical study - apropos of 500 consecutive autopsies. Arch Mal Coeur Vaiss 1994;87:15-21.

\section{KARGER}

Fax +41613061234

E-Mail karger@karger.ch

www.karger.com
(C) 2007 S. Karger AG, Basel

$1015-9770 / 07 / 0245-0480 \$ 23.50 / 0$

Accessible online at:

www.karger.com/ced 
8 Moehring MA, Spencer MP: Power M-mode Doppler (PMD) for observing cerebral blood flow and tracking emboli. Ultrasound Med Biol 2002;28:49-57.

9 Spencer MP, Moehring MA, Jesurum J, Gray WA, Olsen JV, Reisman $\mathrm{M}$ : Power m-mode transcranial Doppler for diagnosis of patent foramen ovale and assessing transcatheter closure. J Neuroimaging 2004; $14: 342-349$.

Dr. Vijay Sharma

Division of Neurology, Department of Medicine

National University Hospital

Singapore 119074 (Singapore)

Tel. +65 6772 2516, Fax +65 68723566

E-Maildrvijay@singnet.com.sg

\section{Cerebrovasc Dis 2007;24:481 \\ DOI: 10.1159/000108925 \\ Reply to the Letter by Sharma and Chan: Is Transcranial Doppler for the Detection of Venous-to-Arterial Circulation Shunts Reproducible?}

\section{S. Sastry, K. Daly, T. Chengodu, C.N. McCollum \\ Department of Academic Surgery, South Manchester University Hospital, Manchester, UK}

We agree with Sharma and Chan [1] that the recent study by Belvis et al. [2] is further evidence that transcranial Doppler (TCD) is a simple, reliable and minimally invasive approach to the diagnosis and quantification of venous-to-arterial circulation shunts (v-aCS).

Our study clearly demonstrated the importance of optimising and then standardising the preparation of microbubbles [3]. We also believe that standardising the provocation tests, particularly the Valsalva manoeuvre, is important in achieving reliable and reproducible results.

In addition to anatomical reasons why the number of bubbles traversing the heart may be higher in the sitting rather than supine position, our experience is that a more stable TCD signal can be maintained in the sitting position $[4,5]$.
We agree that power M-mode TCD may improve the detection and quantification of $\mathrm{v}$-aCS. It may be possible to adapt the criteria for $\mathrm{v}$-aCS used in our study if the number of microbubbles detected by power M-mode TCD can be compared with the number on standard TCD; if power M-mode detects 50\% more microbubbles, our definition of a 'significant' $\mathrm{v}$-aCS would be $\geq 20 \mathrm{mi}$ crobubbles. Whilst a study to compare power M-mode and standard TCD would be worthwhile, we are concerned with Sharma and Chan's suggestion of repetitive testing for $\mathrm{v}$-aCS. There have been 5 case reports of transient ischaemic attack and 1 of stroke following intravenous injection of agitated air and saline during echocardiography $[6,7]$. Our practice is to limit the number tests wherever possible and to stop testing once a large shunt has been demonstrated for safety reasons.

\section{References}

1 Sharma VK, Chan BPL: Comment on 'Is transcranial Doppler for the detection of veous-to-arterial circulation shunts reproducible?' Cerebrovasc Dis 2007;24:480-481.

2 Belvis R, Leta RG, Marti-Fabregas J, Cocho D, Carreras F, Pons-Llado G, et al: Almost perfect concordance between simultaneous transcranial Doppler and transesophageal echocardiography in the quantification of right-to-left shunts. J Neuroimaging 2006;16:133-138.

3 Sastry S, Daly K, Chengodu T, McCollum C: Is transcranial Doppler for the detection of venous-to-arterial circulation shunts reproducible? Cerebrovasc Dis 2007;23:424-429.

4 Sastry S, Riding G, Morris J, Taberner D, Cherry N, Heagerty A, McCollum CN: The role of venous-to-arterial circulation shunts and thrombophilia in myocardial infarction and ischaemic stroke in young adults. J Am Coll Cardiol 2006;48:686-691.

5 Purandare N, Oude Voshaar RC, Burns A, Velupandian UM, McCollum C: Paradoxical embolization: a potential cause of cerebral damage in Alzheimer's disease? Neurol Res 2006;28:679-684.

6 Dittrich HC: Transient ischemic attack after air-contrast echocardiography. Ann Intern Med 1995;123:731-732.

7 Srivastava TN, Undesser EK: Transient ischemic attack after air contrast echocardiography in patients with septal aneurysm. Ann Intern Med 1995;122:396.

Sanjay Sastry

Department of Academic Surgery

South Manchester University Hospital

Southmoor Road, Manchester M23 9PT (UK)

Tel. +44161291 5853, Fax +441612915854

E-Mail cnmcc@manchester.ac.uk 